\title{
Dijet Production in Diffractive DIS and Photoproduction at ZEUS
}

\author{
Yuji Yamazaki, for the ZEUS collaboration \\ Kobe University - Department of Physics, Graduate School of Sciences \\ 1-1 Rokko-dai, Nada, Kobe 657-8501 - Japan
}

\begin{abstract}
Recent ZEUS measurements on dijet production in diffractive deep-inelastic scattering and diffractive photoproduction are reviewed. The measured cross sections are compared to next-to-leading order perturbative QCD calculations using recent diffractive parton densities. For diffractive photoproduction, where the factorisation theorem is not proven, such comparison serves as a test of QCD factorisation. No clear evidence of factorisation breaking was found.
\end{abstract}

\section{Introduction}

Diffractive interactions studied mainly in the HERA ep collisions are so-called photondissociation processes, where the proton scatters with a virtual photon from the electron with the proton remaining intact and the photon dissociating into a multi-hadron state $X$. The exchanged state carries only a small fraction, $x_{\mathbb{P}}$, of the longitudinal momentum of the proton lost through the scatter. The scaling-violation behaviour of semi-inclusive deep-inelastic scattering (DIS) cross sections of the diffractive processes are used to extract the diffractive parton densities ( $\mathrm{dPDFs}$ ), defined as the PDFs of the proton undergoing a diffractive scattering.

Although the quark densities in $\mathrm{dPDF}$ can be obtained precisely from this procedure, gluons are only loosely constrained. The dijet production, both in DIS and photoproduction, are dominated by the boson-gluon fusion process, where a hard collision of virtual photon and a gluon produces a high- $p_{T}$ quark-antiquark pair. The diffractive dijet cross sections, therefore, are more directly sensitive to the gluonic content of the diffractive exchange. The dijet process also allows to reconstruct the momentum fraction, $z_{\mathbb{P}}$, of the initial parton in the diffractive exchange participating in the hard scattering, using the longitudinal momenta of jets: $z_{\mathbb{P}}^{\mathrm{OBS}}=\left(E_{T}^{\text {jet1 }} e^{\eta^{\text {jet1 }}}+E_{T}^{\text {jet2 }} e^{\eta^{\text {jet2 }}}\right) /\left(2 x_{\mathbb{P}} E_{p}\right)$, where $E_{T}^{\text {jet1 }}$ and $E_{T}^{\text {jet2 }}$ are the transverse energy of the jets with the highest and second highest $E_{T}^{\text {jet }}$, respectively, $\eta^{\text {jet1 }}$ and $\eta^{\text {jet2 }}$ are the pseudorapidities of the corresponding jets and $E_{p}$ is the energy of the incoming proton.

The QCD factorisation theorem is proven for diffractive DIS with a presence of a large photon virtuality $Q^{2}$ : the dijet cross sections in diffractive DIS can also be expressed as a convolution of the dPDFs and coefficient functions. No such proof exists for diffractively photoproduced dijets. In fact, such factorisation appears to fail at the Tevatron, the highest energy $p \bar{p}$ collisions: the cross sections were suppressed with respect to the pQCD calculations. This is believed to be attributed to a class of events with more than one parton-parton scatter between the proton and the diffractive exchange, destroying the diffractive conditions and thus breaking the factorisation. A similar phenomenon could occur in photoproduction events. The photon can resolve into more than one partons and thus could also suppress the diffractive cross sections of the resolved photon processes. For dijet events, the resolved processes can be identified with an estimator, $x_{\gamma}^{\mathrm{OBS}}$, of the momentum fraction, $x_{\gamma}$, of the parton in the photon participating in the hard scattering. The estimator is reconstructed again using the longitudinal momenta of the jets: $x_{\gamma}^{\mathrm{OBS}}=\left(E_{T}^{\mathrm{jet} 1} e^{-\eta^{\mathrm{jet} 1}}+E_{T}^{\mathrm{jet} 2} e^{-\eta^{\mathrm{jet} 2}}\right) /\left(2 y E_{e}\right)$, where 
$y$ is the fraction of the energy of colliding photons to the incoming electron energy $E_{e}$. Events with low values of $x_{\gamma}^{\mathrm{OBS}}$ are dominated by resolved processes.

\section{Dijets in diffractive DIS}

Figure 1 shows the preliminary measurement of the differential cross sections for diffractive DIS processes by ZEUS[2]. The measurement was performed for $5<Q^{2}<100 \mathrm{GeV}^{2}, 100<$ $W<250 \mathrm{GeV}$, the centre-ofmass energy of the virtual photon and the proton, $x_{\mathbb{P}}<0.03$, $E_{T}^{\mathrm{jet} 1}>5 \mathrm{GeV}$ and $E_{T}^{\mathrm{jet} 2}>4 \mathrm{GeV}$. The jets are found in the $\gamma^{*} p$ frame, the centre-of-mass frame of the virtual photon and the proton, using the longitudinallyinvariant $k_{T}$-algorithm. The cross sections are reasonably well described by the theoretical calculations at the next-to-leading or$\operatorname{der}(\mathrm{NLO})$ by DISENT [3] using the H1 fit 2002(prel.)[4] and ZEUS LPS+charm parameterisations of dPDFs [5]. The H1 fit 2002 dPDFs was extracted from the $\mathrm{H} 1$ measurements of the inclusive diffraction cross sections. The ZEUS
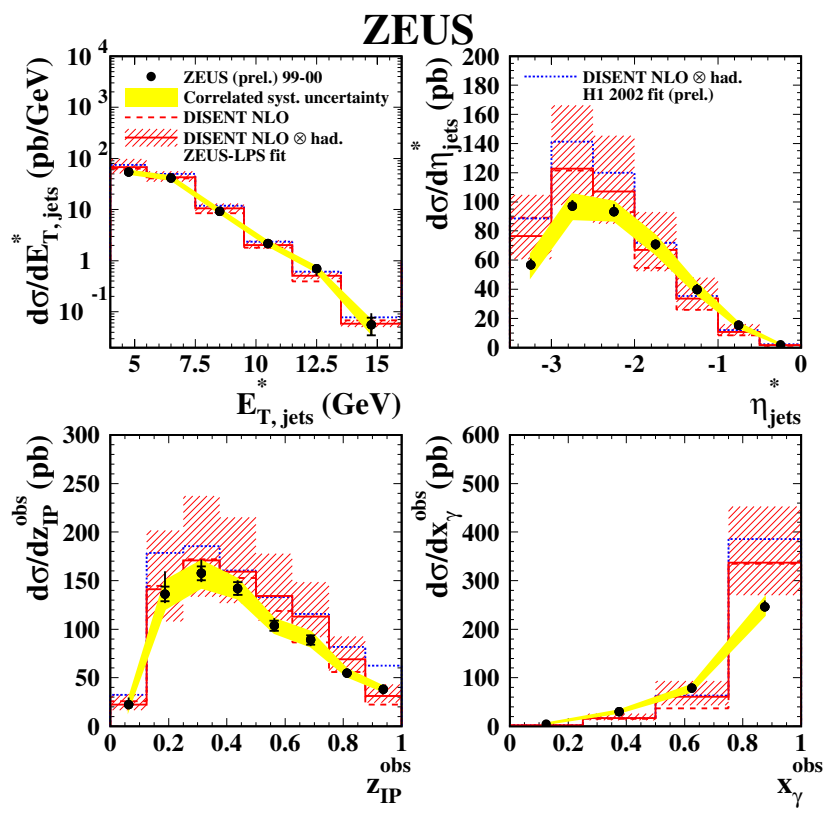

Figure 1: Differential dijet cross sections in the DIS regime for $E_{T}^{\text {jet }}, \eta^{\text {jet }}, z_{\mathbb{P}}^{\mathrm{OBS}}$ and $x_{\gamma}^{\mathrm{OBS}}$, compared to NLO calculations using H1 fit 2002(prel.) and ZEUS LPS+charm as dPDFs.

LPS+charm dPDFs utilised also the charm production cross sections for constraining the dPDFs for gluons. The good agreement shows that both dPDFs are appropriate for estimating other diffractive cross sections.

\section{Dijets in diffractive photoproduction}

The final cross section measurements of the diffractive dijet in photoproduction is presented in this workshop, with the kinematic range of $Q^{2}<1 \mathrm{GeV}^{2}, 0.2<y<0.85, x_{\mathbb{P}}<0.025$, $E_{T}^{\text {jet1 }}>7.5 \mathrm{GeV}$ and $E_{T}^{\mathrm{jet} 2}>6.5 \mathrm{GeV}$. The jets are found using the $k_{T}$ algorithm in the laboratory frame of the HERA beams, $E_{e}=920 \mathrm{GeV}$ and $E_{e}=27.5 \mathrm{GeV}$.

The cross sections are compared to the NLO calculations by Klasen and Kramer [7]. The calculations were originally published with the H1 fit 2002(prel.) dPDFs. The ZEUS collaboration has implemented the new dPDFs, such as the H1 fit 2006 [8], using their program. The result of the calculation, however, show about $10 \%$ difference in both shape and normalisation when compared to the calculation performed by the H1 collaboration using the program by Frixione and Ridolfi [9] in the same kinematic range of the H1 measurement. 


\section{ZEUS}
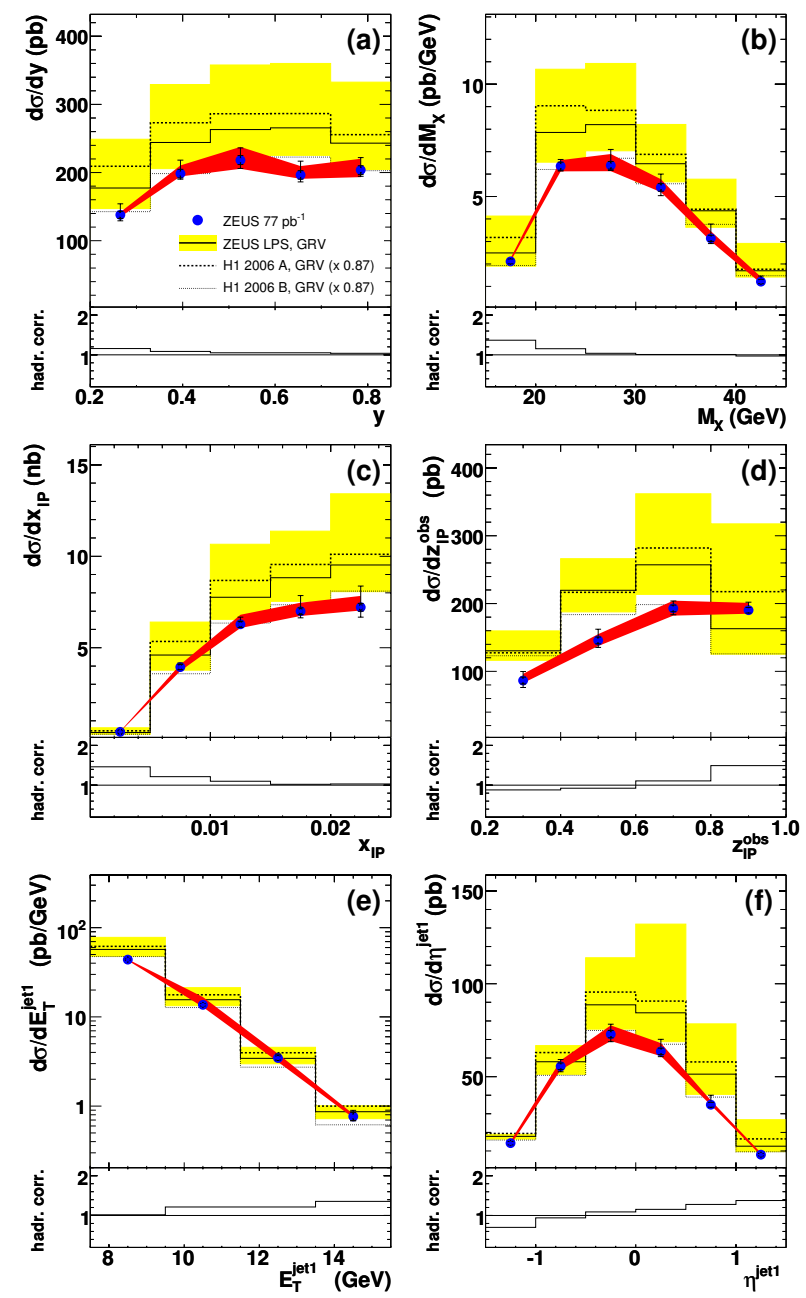

Figure 2: Differential dijet cross sections in the photoproduction regime as a function of (a) $y$, (b) $M_{X}$, (c) $x_{\mathbb{P}}$, (d) $z_{\mathbb{P}}^{\mathrm{OBS}},(\mathrm{e}) E_{T}^{\text {jet1 }}$ and (f) $\eta^{\text {jet1 }}$, compared to NLO calculations using H1 fit $2006 \mathrm{~A}$ and B, and ZEUS LPS+charm as dPDFs.

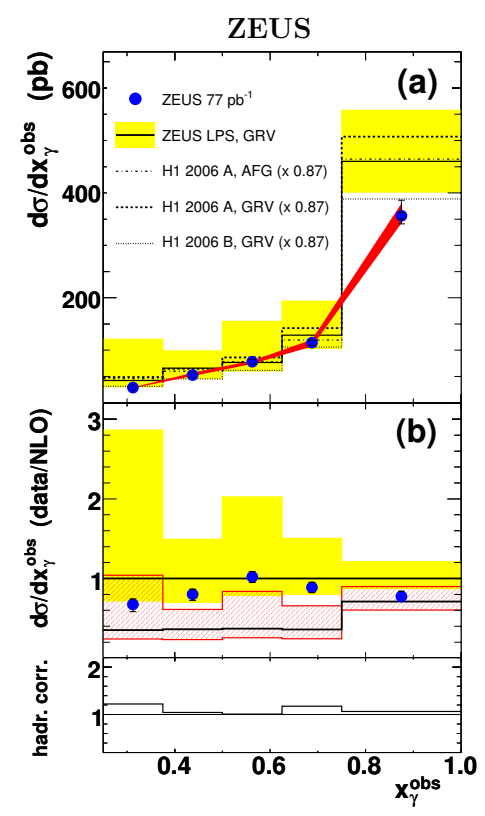

Figure 3: (a) Differential dijet cross sections in the photoproduction regime as a function of $x_{\gamma}^{\mathrm{OBS}}$, compared to NLO calculations using $\mathrm{H} 12006 \mathrm{fit} \mathrm{A}$ and fitB and ZEUS LPS+charm as dPDFs. (b) The ratio of the cross sections to the prediction using the ZEUS LPS+charm dPDFs. The histogram with a hatched band shows the prediction with the resolved photon suppressed according to the prediction by Kaidalov et al. [10]: see text.

This issue is under investigation. The conclusion in this contribution, therefore, is based on the assumption that the NLO calculation is correct within about $10 \%$.

Figure 2 shows the cross sections for the entire range of $x_{\gamma}^{\mathrm{OBS}}$ in comparison to the NLO calculations using the ZEUS LPS parton density as well as two variation of the H1 fit 2006 $\mathrm{dPDF}$, fitA and fitB, which use different assumption on the shape of the gluon density. The cross sections are well described by using the H1 2006 fitB. The ZEUS LPS and H1 2006 
fitA give higher cross sections than the data by about $20 \%$, but agree with the data within theoretical uncertainties.

The $x_{\gamma}^{\mathrm{OBS}}$ dependence of the cross sections are compared to the NLO calculations in Fig. 3. The measurement show good agreement with the calculations ${ }^{\mathrm{a}}$. The model by Kaidalov et al. [10], tuned to explain the suppression of the diffractive dijet production measured by CDF [11], predicts that the resolved photon contribution is suppressed by about $1 / 3$ at HERA. The model with such a rescaling on the resolved processes fails to describe the data. The cross sections for resolved-enriched $\left(x_{\gamma}^{\mathrm{OBS}}<0.75\right)$ and direct-enriched $\left(x_{\gamma}^{\mathrm{OBS}}>0.75\right)$ samples show also good agreement with the NLO calculations using the $\mathrm{H} 1$ 2006 fitB parameterisation (not shown).

Summarising, no clear evidence of factorisation breaking was observed in the diffractive dijet photoproduction at HERA within the experimental and theoretical uncertainties.

\section{Discussion}

The negative observation of factorisation breaking in photoprduction by ZEUS appears to conflict with the conclusion from the corresponding measurement by the H1 collaboration [12]. The $\mathrm{H} 1$ data, however, starts from lower $E_{T}^{\text {jet }}: E_{T}^{\text {jet1 }}>5 \mathrm{GeV}$ and $E_{T}^{\text {jet2 }}>4 \mathrm{GeV}$. The measurement is also extended to $x_{\mathbb{P}}<0.03$, higher than ZEUS. For both H1 and ZEUS measurements, the $E_{T}^{\text {jet }}$ dependence is not well reproduced by NLO: the cross sections at low $E_{T}^{\text {jet }}$ tend to be overestimated by the calculation (see Fig. 2e). This might have lead different conclusions among measurements depending on the $E_{T}^{\text {jet }}$ range. A more direct comparison with the same kinematic range may help to have a definitive conclusion on this issue.

\section{References}

[1] Slides: http: //indico. cern. ch/contributionDisplay $\cdot$ py? contribId=63\&sessionId $=7 \&$ conf $I d=9499$

[2] ZEUS Coll., Contrib. 342 for the EPS-HEP2005 Conf.(unpublished).

[3] S. Catani and M.H. Seymour, Nucl. Phys. B485, 291 (1997); Erratum-ibid. B510, 503 (1997).

[4] H1 Coll., Contrib. 980 for the ICHEP2002 Conf.(unpublished).

[5] ZEUS Coll., S. Chekanov et al., Eur. Phys. J. C38, 43 (2004).

[6] ZEUS Coll., Contrib. 249 for the ICHEP2004 Conf.(unpublished).

[7] M. Klasen and G. Kramer, Eur. Phys. J. C38, 93 (2004).

[8] H1. Coll., A. Aktas et al., Eur. Phys. J. C48, 715 (2006).

[9] S. Frixione, Z. Kunszt and A. Signer, Nucl. Phys. B467, 399 (1996); S. Frixione, Nucl. Phys. B507, 295 (1997).

[10] A.B. Kaidalov et al., Eur. Phys. J. C21, 521 (2001); A.B. Kaidalov et al., Phys. Lett. B567, 61 (2003);

[11] CDF Coll., T. Affolder et al., Phys. Rev. Lett. 84, 5043 (2000).

[12] H1 Coll., A. Aktas et al., DESY-07-018, Submitted to Eur. Phys. J. C.

aThis statement is somewhat different from what was presented in the preliminary presentation by the ZEUS collaboration at the ICHEP2004 conference[6], where the cross sections as a function of $x_{\gamma}^{\mathrm{OBS}}$ agreed with the NLO in shape but with a normalisation factor of about 0.6. Three issues have lead the change in the relation between the measurement and the calculation: (1) the diffractive parton densities was the H1 fit 2002(prel.) parameterisation, which tend to give a higher cross section than the H1 2006 fitB; (2) the subtraction of the proton dissociation from the H1 dPDFs was necessary since the ZEUS dijet cross section is corrected to the elastic-proton photon dissociation $e p \rightarrow e p X$. The H1 dPDFs are therefore scaled down by $13 \%$ in this presentation, which was missing in the ICHEP2004 contribution; (3) The ZEUS cross section measurements are revised and have changed within the quoted systematic uncertainties of the ICHEP2004 contribution. 